\title{
Percutaneous patent foramen ovale closure for cryptogenic stroke: learning from clinical trial and error
}

\author{
Mohammad K. Mojadidi, Ahmed N. Mahmoud, Islam Y. Elgendy \\ Division of Cardiology, Department of Medicine, University of Florida College of Medicine, Gainesville, Florida, USA \\ Correspondence to: Islam Y. Elgendy, MD. 1600 SW Archer Road, PO Box 100277, Gainesville, FL 32610, USA. Email: iyelgendy@gmail.com.
}

Submitted Sep 21, 2017. Accepted for publication Sep 25, 2017.

doi: $10.21037 /$ jtd.2017.09.153

View this article at: http://dx.doi.org/10.21037/jtd.2017.09.153

Each year, over 690,000 people suffer from ischemic stroke in the United States. Of these, $25-30 \%$ are cryptogenic where the etiology cannot be determined (1). In the late 1980 s, observational studies suggested a higher prevalence of patent foramen ovale (PFO) $(40-50 \%$ versus $10-15 \% ; \mathrm{P}<0.001)$ in young people aged $<40-55$ years with cryptogenic stroke $(2,3)$. Contrary to subsequent non-randomized studies (4), the first three randomized clinical trials (CLOSURE I, PC and RESPECT) failed to demonstrate superiority of percutaneous PFO closure over medical therapy for secondary prevention of stroke in a primary analysis (5-7).

The CLOSURE I trial randomized patients to transcatheter PFO closure with the STARFlex device (NMT Medical, Boston, Massachusetts, USA) or medical therapy for secondary prevention of stroke or transient ischemic attack (TIA). Inconsistency between prior observational studies and CLOSURE I has been attributed to the device itself, which paradoxically had a high rate of thrombogenesis, atrial fibrillation, and residual right-toleft shunting post-closure $(5,8)$. The PC trial randomized patients who had cryptogenic embolism and a PFO to medical therapy or closure with the Amplatzer PFO occluder (Abbott; Chicago, Illinois, USA). Despite that the study utilized a device which had a low rate of atrial fibrillation, thrombosis and residual shunting post-closure, the trial demonstrated a non-significant trend $(\mathrm{P}>0.05)$ favoring PFO closure (6). Discrepancy between the PC trial and previous observational studies has been attributed to the study being significantly underpowered with a high risk of type II error. In addition, inclusion of patients with TIA and other peripheral embolism was not reflective of the study population included in most observational studies. Lack of blinding may also have potentiated the use of off-label $\mathrm{PFO}$ occluding devices in patients included in the medical therapy arm $(9,10)$. Although the RESPECT trial did not demonstrate superiority of closure using the Amplatzer device in an intention-to-treat analysis (7), the extended follow-up data (median 5.9 years) showed superiority of PFO closure with a $62 \%$ relative risk reduction in recurrent cryptogenic stroke; the benefit of closure was enhanced in those with a large shunt or atrial septal aneurysm. The reduced risk of recurrent stroke in the closure arm was observed despite inclusion of three patients who did not receive a device. There was no difference in overall serious adverse events (SAE) between the two groups (11). Another important limitation was the slow recruitment of patients in these randomized trials (e.g., it took approximately 8 years to recruit patients in the RESPECT trial), which may partly be explained by patients' reluctance to undergo randomization, due to their personal preference for medical therapy or closure.

A patient level meta-analysis of CLOSURE I, PC and RESPECT trials further corroborated that percutaneous $\mathrm{PFO}$ closure is superior to medical therapy for reducing risk of recurrent stroke in patients with cryptogenic stroke (12). Following success of the RESPECT trial, the Food and Drug Administration approved the Amplatzer PFO occluder for transcatheter PFO closure to reduce risk of recurrent ischemic stroke in patients with cryptogenic stroke, attributed to paradoxical embolism by a neurologist and cardiologist (13).

Since then, two new randomized controlled trials, CLOSE and Gore-REDUCE, have been completed $(14,15)$. Among patients randomized in the CLOSE trial, no stroke occurred in the device arm. Closure proved superior to 


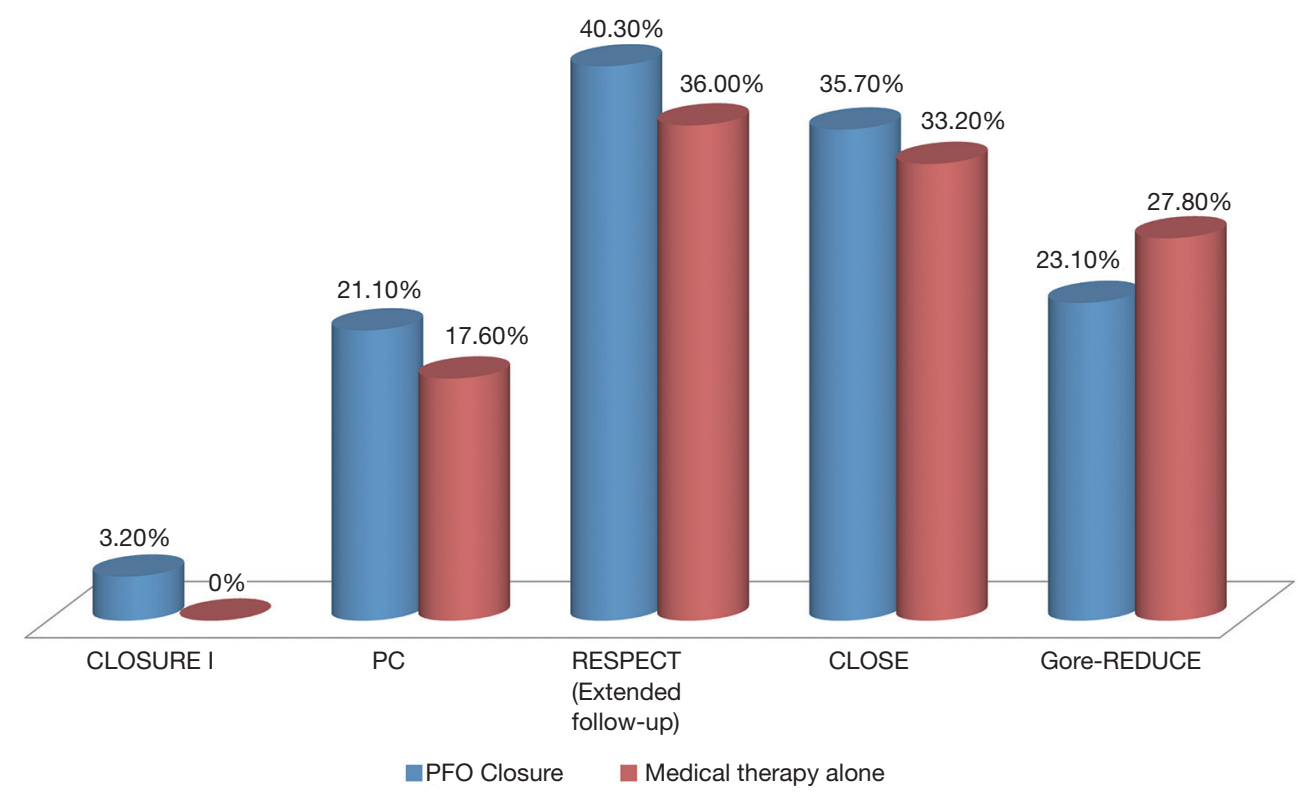

Figure 1 Rate of overall SAEs in each clinical trial comparing transcatheter PFO closure to medical therapy in patients with cryptogenic stroke. SAE was defined by CLOSURE I as major adverse device-related events resulting in surgical intervention to repair a PFO after study treatment, recovery of an embolized device at a subsequent cardiac surgery, placement of a pacemaker resulting from PFO closure, thrombus on the device that did not adequately resolve with pharmacotherapy, or repair of a damaged coronary vessel or cardiac structure as a result of device implantation. PC defined SAE as any adverse events leading to hospital admission or prolongation of hospitalization, life-threatening events or death. RESPECT and CLOSE defined SAE as any events that resulted in death, required either inpatient hospitalization or prolongation of hospitalization, were life-threatening, resulted in a persistent or significant disability/incapacity or resulted in a congenital anomaly/birth defect. Gore-REDUCE defined SAE as any major events attributed to having a relationship to the device, procedure, antiplatelet or unknown cause. SAE, serious adverse events; PFO, patent foramen ovale.

antiplatelet therapy for stroke prevention $(0 \%$ versus $6.0 \%$, HR $0.03 ; 95 \%$ CI, $0-0.26 ; \mathrm{P}<0.001)$ at a mean followup of $5.3 \pm 2.0$ years. Similarly, the Gore-REDUCE trial demonstrated reduced risk of recurrent stroke in the device $\operatorname{arm}(1.4 \%$ versus $5.4 \%$, HR 0.23 ; 95\% CI, 0.09-0.62; $\mathrm{P}=0.002)$ at a median follow-up of 3.2 years. While the two trials reported no difference in SAEs and bleeding between the closure and medical therapy groups, both had a higher incidence of atrial arrhythmias in the device $\operatorname{arm}(\mathrm{P}<0.05)$.

Reasons for disagreement between the earlier negative trials and the newer positive trials may be attributed to selective inclusion of patients who had a stroke that is more likely due to paradoxical embolism. The GoreREDUCE trial used strict omission criteria to exclude other causes of stroke such as large-artery atherosclerotic disease and small-vessel disease (lacunar infarcts) through extensive cerebrovascular imaging and exclusion of uncontrolled risk factors. CLOSE only included patients with an atrial septal aneurysm or large right-to-left shunt.
Thus, appropriate patient selection by recommending device closure for those who underwent extensive exclusion of other causes of stroke is crucial. Although percutaneous PFO closure has been shown to carry a very small risk of SAEs in the clinical trials (Figure 1), it is important to engage patients in the conversation when considering closure.

A study level meta-analysis of all five randomized trials confirmed the lower risk of recurrent stroke with transcatheter PFO closure (2.2\% versus $4.0 \%$; RR 0.54 , $\mathrm{P}=0.02$ ) at a mean follow-up of 2.9 years (Figure 2). The meta-analysis found a four-fold increased risk of atrial fibrillation in the device group. Post-closure atrial fibrillation is a real concern and found to be device dependent (Gore devices $>$ STARFlex device $>$ Amplatzer PFO occluder) (16). However, most cases of atrial fibrillation in the trials were an early single paroxysm occurring and resolving spontaneously or with cardioversion.

In conclusion, lessons learned from earlier clinical trials 


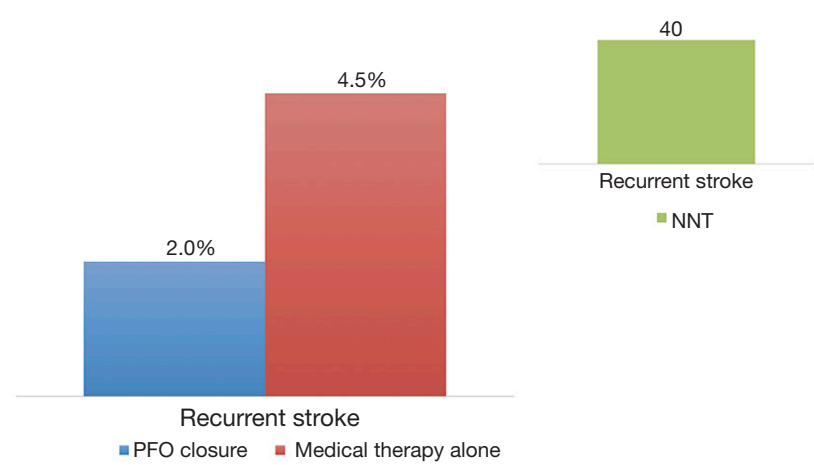

Figure 2 Risk of recurrent stroke in all clinical trials comparing transcatheter PFO closure to medical therapy in patients with cryptogenic stroke. NNT, number needed to treat; PFO, patent foramen ovale.

have allowed us to identify a more "pure" patient population at highest risk of recurrent paradoxical embolism. Stringent exclusion criteria to rule out other causes of stroke are crucial to identifying patients who truly have stroke that is cryptogenic. Results from the newer trials correlate with our understanding that higher risk patients (i.e., presence of large right-to-left shunt or atrial septal aneurysm) receive the most benefit from closure. We call for a change in guidelines to recommend percutaneous PFO closure as first line therapy for all patients aged $\leq 60$ years old with cryptogenic ischemic stroke, especially for the subgroup at greatest risk of recurrent paradoxical embolism. Given the concern of post-closure atrial fibrillation, we additionally recommend prolonged cardiac monitoring ( $\geq 30$ days) to more aggressively exclude patients with undiagnosed atrial fibrillation. Although prolonged cardiac monitoring is found to be superior compared with standard 24-hour monitoring, to detect atrial fibrillation in patients with stroke that is thought to be cryptogenic $(17,18)$, this was not an inclusion criterion in any of the PFO and stroke trials. Future research should focus on the longterm prognostic implications of atrial fibrillation post-device implant, which continue to be unknown.

\section{Acknowledgements}

None.

\section{Footnote}

Conflicts of Interest: The authors have no conflicts of interest to declare.

\section{References}

1. Benjamin EJ, Blaha MJ, Chiuve SE, et al. Heart disease and stroke statistics-2017 update: a report from the American Heart Association. Circulation 2017;135:e229-e445.

2. Lechat P, Mas JL, Lascault G, et al. Prevalence of patent foramen ovale in patients with stroke. N Engl J Med 1988;318:1148-52.

3. Webster MW, Chancellor AM, Smith HJ, et al. Patent foramen ovale in young stroke patients. Lancet 1988;2:11-2.

4. Agarwal S, Bajaj NS, Kumbhani DJ, et al. Meta-analysis of transcatheter closure versus medical therapy for patent foramen ovale in prevention of recurrent neurological events after presumed paradoxical embolism. JACC Cardiovasc Interv 2012;5:777-89.

5. Furlan AJ, Reisman M, Massaro J, et al. Closure or medical therapy for cryptogenic stroke with patent foramen ovale. N Engl J Med 2012;366:991-9.

6. Meier B, Kalesan B, Mattle HP, et al. Percutaneous closure of patent foramen ovale in cryptogenic embolism. N Engl J Med 2013;368:1083-91.

7. Carroll JD, Saver JL, Thaler DE, et al. Closure of patent foramen ovale versus medical therapy after cryptogenic stroke. N Engl J Med 2013;368:1092-100.

8. Mojadidi MK, Gevorgyan R, Tobis J. Device Closure of Patent Foramen Ovale or Medical Therapy for Cryptogenic Stroke: The CLOSURE I Trial. In: Amin Z, Tobis J, Sievert H, et al. editors. Springer London: Patent Foramen Ovale, 2015:173-9

9. Khattab AA, Meier B. The PC Trial: An Effective Treatment Not Demonstrating Effective Power. In: Amin Z, Tobis J, Sievert H, et al. editiors. Springer London: Patent Foramen Ovale, 2015:185-8.

10. Mojadidi MK, Christia P, Salamon J, et al. Patent foramen ovale: Unanswered questions. Eur J Intern Med 2015;26:743-51.

11. Saver JL, Carroll JD, Thaler DE, et al. Long-Term Outcomes of Patent Foramen Ovale Closure or Medical Therapy after Stroke. N Engl J Med 2017;377:1022-32.

12. Kent DM, Dahabreh IJ, Ruthazer R, et al. Device Closure of Patent Foramen Ovale After Stroke: Pooled Analysis of Completed Randomized Trials. J Am Coll Cardiol 2016;67:907-17.

13. AMPLATZER PFO Occluder - P120021.

Available online: https://www.fda.gov/

MedicalDevices/ProductsandMedicalProcedures/ DeviceApprovalsandClearances/Recently- 
ApprovedDevices/ucm526921.htm

14. Mas JL, Derumeaux G, Guillon B, et al. Patent Foramen Ovale Closure or Anticoagulation vs. Antiplatelets after Stroke. N Engl J Med 2017;377:1011-21.

15. Søndergaard L, Kasner SE, Rhodes JF, et al. Patent Foramen Ovale Closure or Antiplatelet Therapy for Cryptogenic Stroke. N Engl J Med 2017;377:1033-42.

16. Mojadidi MK, Elgendy AY, Elgendy IY, et al. Transcatheter patent foramen ovale closure after cryptogenic stroke:

Cite this article as: Mojadidi MK, Mahmoud AN, Elgendy IY. Percutaneous patent foramen ovale closure for cryptogenic stroke: learning from clinical trial and error. J Thorac Dis 2017;9(11):4222-4225. doi: 10.21037/jtd.2017.09.153
An updated meta-analysis of randomized trials. JACC Cardiovasc Interv 2017. doi: 10.1016/j.jcin.2017.09.002

17. Sanna T, Diener HC, Passman RS, et al. Cryptogenic stroke and underlying atrial fibrillation. N Engl J Med 2014;370:2478-86.

18. Gladstone DJ, Spring M, Dorian P, et al. Atrial fibrillation in patients with cryptogenic stroke. $\mathrm{N}$ Engl J Med 2014;370:2467-77. 